\title{
A CLASS OF NONLINEAR VARIATIONAL INEQUALITIES INVOLVING PSEUDOMONOTONE OPERATORS
}

\author{
RAM U. VERMA \\ International Publications \\ 12046 Coed Drive \\ Orlando, FL 32826 USA
}

(Received June, 1998; Revised January, 1999)

We present the solvability of a class of nonlinear variational inequalities involving pseudomonotone operators in a locally convex Hausdorff topological vector spaces setting. The obtained result generalizes similar variational inequality problems on monotone operators.

Key words: Nonlinear Variational Inequality, Pseudomonotone Operator, Locally Convex Space, Monotone Operator.

AMS subject classifications: $49 \mathrm{~J} 40$.

\section{Introduction}

Recently, the author [4] studied a class of nonlinear variational inequalities in a locally convex Hausdorff topological vector space setting and extended a class of nonlinear variational inequalities involving monotone operators by utilizing a fixed point theorem [5]. The aim of this note is to present a class of generalized nonlinear variational inequalities involving pseudomonotone operators in a locally convex Hausdorff topological vector space setting. The obtained result generalizes the variational inequality results involving monotone operators in a similar setting, especially [4,5]. For more details on variational and hemivariational inequalities in a Hilbert space setting, we refer to [3].

Let $K$ be a subset of a real locally convex Hausdorff topological vector space $X$ with its dual $X^{\prime}$ and let $T: K \rightarrow X^{\prime}$ be a pseudomonotone nonlinear mapping. Let $\langle w, x\rangle$ denote the duality pairing between the elements $w$ in $X^{\prime}$ and elements $x$ in $X$. We consider the nonlinear variational inequality (NVI) problem: Find an element $u_{0}$ in $K$ such that

$$
\left\langle T u_{0}, v-u_{0}\right\rangle \geq 0 \text { for all } v \text { in } K .
$$

A mapping $T: K \rightarrow X^{\prime}$ is said to be:

(i) pseudomonotone [1] if 


$$
\langle T u, v-u\rangle \geq 0 \Rightarrow\langle T v, v-u\rangle \geq 0 \text { for all } u, v \text { in } K
$$

(ii) quasimonotone [1] if

$$
\langle T u, v-u\rangle>0=\Rightarrow\langle T v, v-u\rangle \geq 0 \text { for all } u, v \text { in } K \text {; }
$$

(iii) monotone if for all $u, v$ in $K$, we have

$$
\langle T v-T u, v-u\rangle \geq 0 .
$$

We note that the following implication holds:

$$
\text { monotone } \Rightarrow \text { pseudomonotone } \Rightarrow \text { quasimonotone. }
$$

Before we consider our main result, we need to recall an auxiliary result (a variant of [5, Proposition 9.9] and similar to [2, Theorem 1] crucial to the NVI problem (1) at hand.

Lemma 1.1: Let $K$ be a nonempty, compact and convex subset of a locally convex space $X$ and let $S: K \rightarrow P(K)$ be a multivalued mapping such that the following assumptions hold:

(i) The set $S(x)$ is nonempty and convex for all $x \in K$.

(ii) The preimages $S^{-1}(y)$ contain relatively open subsets $U_{y}$ with respect to $K$ for all $y$ in $K$.

(iii) $K=\bigcup\left\{U_{y}: y \in K\right\}$.

Then there exists an element $x_{0}$ in $K$ such that $x_{0} \in S\left(x_{0}\right)$.

\section{The Main Result}

This section deals with the main result on the solvability of the NVI problem (1).

Theorem 2.1: Let $K$ be a nonempty, compact and convex subset of a locally convex Hausdorff topological vector space $X$ and let $T: K \rightarrow X^{\prime}$ be a pseudomonotone mapping. Then the NVI problem (1) has a solution $u_{0}$ in $K$.

Proof: Assume the NVI problem (1) has no solution. Then for each $u \in K$, the set $\{v$ in $K:\langle T u, v-u\rangle<0\}$ is nonempty. As a result, if we define a multivalued mapping $F: K \rightarrow P(K)$ by

$$
F(u)=\{v \text { in } K:\langle T u, v-u\rangle<0\}
$$

then clearly $F(u)$ is nonempty and convex for each $u$ in $K$. It follows that

$$
F^{-1}(v)=\{u \in K: v \in F(u)\}=\{u \in K:\langle T u, v-u\rangle<0\} .
$$

It suffices to show that $F^{-1}(v)$ is relatively open with respect to $K$. For each $v$ in $K$, the complement of $F^{-1}(v)$ in $K$,

$$
c_{K}\left(F^{-1}(v)\right)=\{u \in K:\langle T u, v-u\rangle \geq 0\}
$$




$$
\subset\{u \in K:\langle T v, v-u\} \geq 0\}
$$

by the pseudomonotonicity of $T$, denoted by $G(v)$. We need to show that $G(v)$ is convex and closed in $K$. To show $G(v)$ is convex, if $u_{1}, u_{2} \in G(v), 0<t<1$ and $u_{t}=t u_{1}+(1-t) u_{2}$, then $u_{t} \in K$ and, $\left\langle T v, v-u_{1}\right\rangle \geq 0$ and $\left\langle T v, v-u_{2}\right\rangle \geq 0$. As a result of this, we have

$$
\begin{gathered}
\left\langle T v, v-u_{t}\right\rangle=\left\langle T v, v-t u_{1}-(1-t) u_{2}\right\rangle=\left\langle T v, t\left(v-u_{1}\right)+(1-t)\left(v-u_{2}\right)\right\rangle \\
=t\left\langle T v, v-u_{1}\right\rangle+(1-t)\left\langle T v, v-u_{2}\right\rangle \geq 0
\end{gathered}
$$

This implies $u_{t} \in G(v)$. Next, to show $G(v)$ is relatively closed with respect to $K$, let $\left\{u_{a}\right\}$ be a Moore-Smith sequence in $G(v)$. Then $\left\langle T v, v-u_{a}\right\rangle \geq 0$. Assume $u_{a} \rightarrow u$ in $K$. Now all we need is to show $u \in G(v)$. We can now express

$$
\begin{gathered}
\langle T v, v-u\rangle=\left\langle T v, v-u_{a}\right\rangle+\left\langle T v, u_{a}-u\right\rangle \\
\geq 0+\left\langle T v, u_{a}-u\right\rangle=0 .
\end{gathered}
$$

Thus, $u \in G(v)$. Since $G(v)$ is a closed convex subset of $K$, it implies $c_{K}(G(v))=$ $\{u \in K:\langle T v, v-u\rangle<0\}$ is open in $K$. This results in

$$
c_{K}(G(v)) \subset F^{-1}(v) \text { since any } u \notin F^{-1}(v) \text { implies } u \notin c_{K}(G(v)) .
$$

Hence, for each $v$ in $K, F^{-1}(v)$ contains an open subset $c_{K}(G(v))$. Thus for all $u$ in $K$, there exists an element $v$ in $K$ such that $\langle T v, v-u\rangle<0$. Therefore, $K=$ $\bigcup\left\{c_{K}(G(v)): v \in K\right\}$. Now by Lemma 1.1, there exists an element $x_{0}$ in $K$ such that $x_{0} \in F\left(x_{0}\right)$, that means $0>\left\langle T x_{0}, x_{0}-x_{0}\right\rangle=0$, a contradiction. This completes the proof.

Corollary 2.1: Let $X$ be a locally convex Hausdorff topological vector space and $K$ a nonempty, compact and convex subset of $X$. Suppose that $T: K \rightarrow X^{\prime}$ (dual of $X$ ) is a monotone mapping. Then the NVI problem (1) has a solution.

\section{References}

[1] Karamardian, S., Complementarity problems over cones with monotone and pseudomonotone maps, J. Optimiz. Theor. Appl. 18 (1970), 445-454.

[2] Tarafdar, E., On some nonlinear variational inequalities, Proc. AMS 67 (1977), 95-98.

[3] Verma, R.U., Nonlinear variational and constrained hemivariational inequalities involving relaxed operators, ZAMM 77:5 (1997), 387-391.

[4] Verma, R.U., Strongly monotone nonlinear variational inequalities, Math. Sci. Res. Hot-Line 2:2 (1998), 21-24.

[5] Zeidler, E., Nonlinear Functional Analysis and its Applications I, SpringerVerlag, New York 1986. 


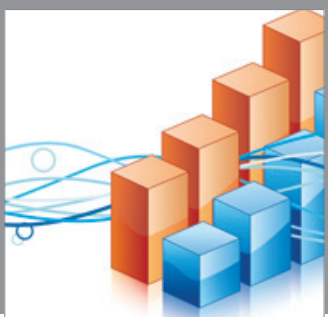

Advances in

Operations Research

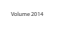

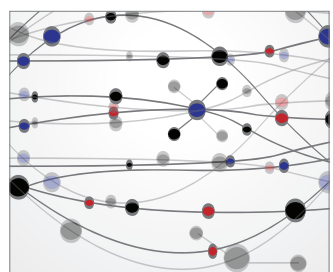

\section{The Scientific} World Journal
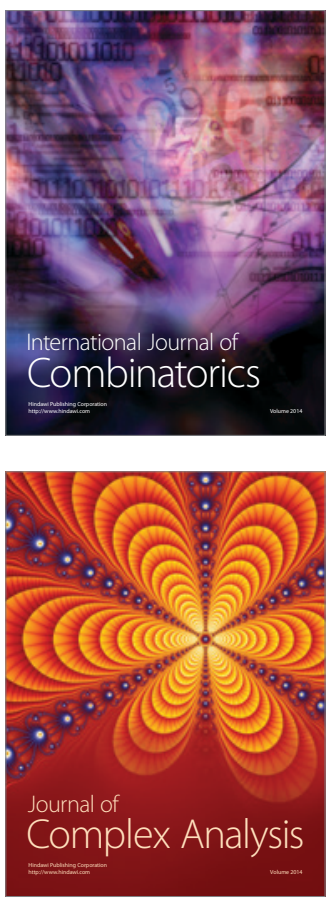

International Journal of

Mathematics and

Mathematical

Sciences
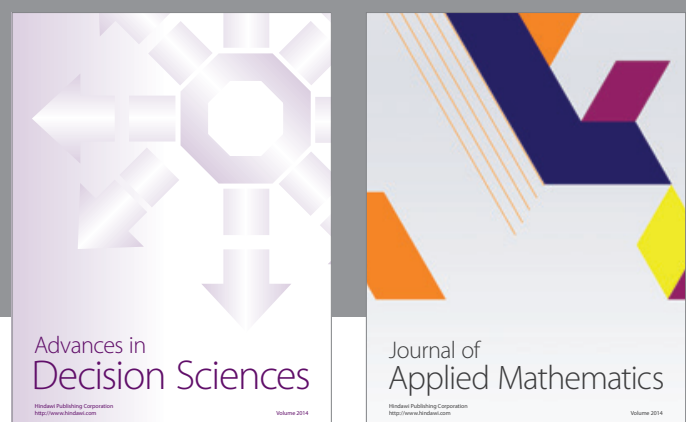

Journal of

Applied Mathematics
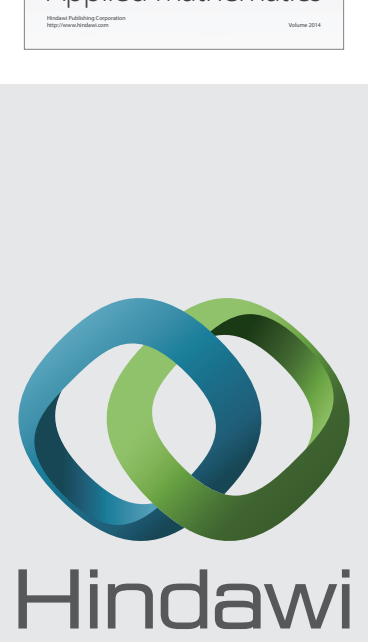

Submit your manuscripts at http://www.hindawi.com
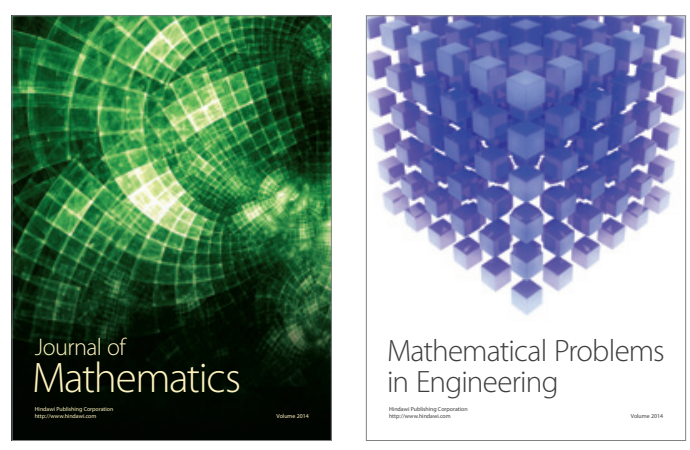

Mathematical Problems in Engineering
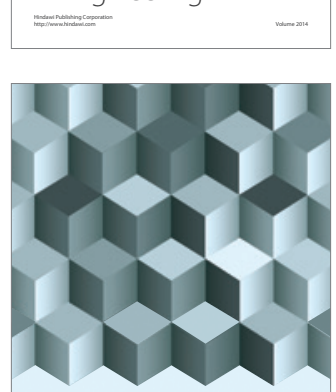

Journal of

Function Spaces
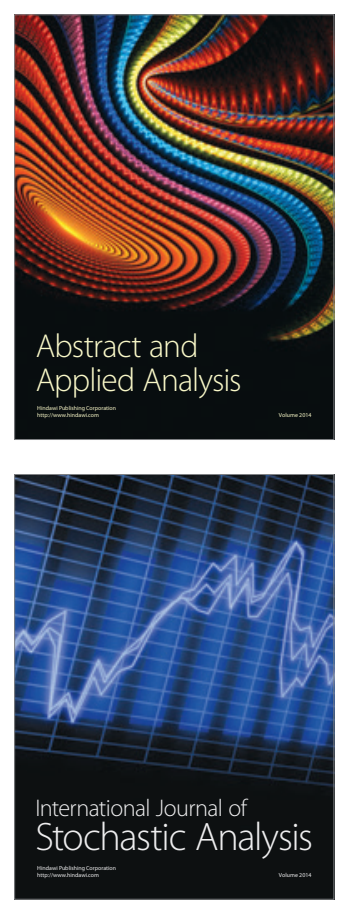

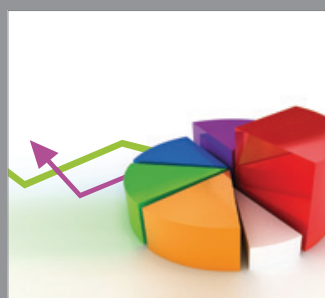

ournal of

Probability and Statistics

Promensencen
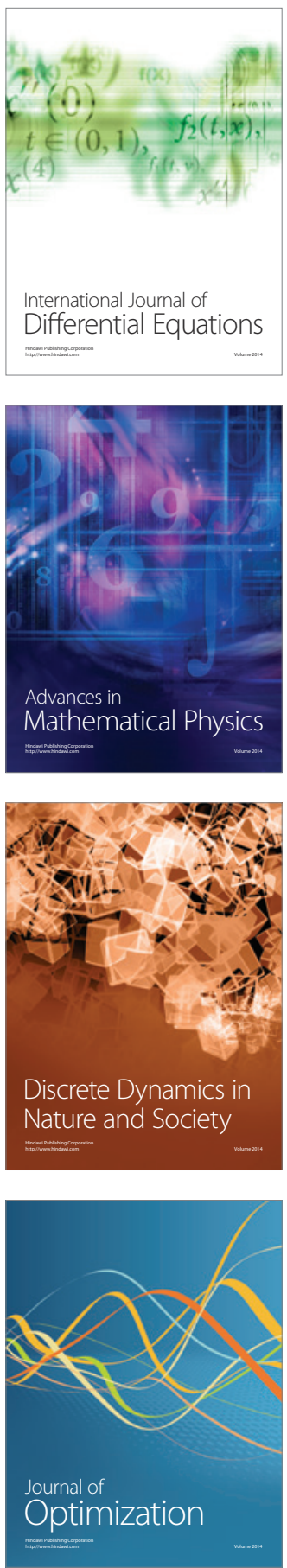